\title{
Dietary patterns are associated with various vascular health markers and complications in type 1 diabetes
}

\author{
Ahola, Aila J.
}

2016-08

Ahola , A J , Freese , R , Mäkimattila , S , Forsblom , C , Groop , P-H \& FinnDiane Study Grp 2016 , ' Dietary patterns are associated with various vascular health markers and complications in type 1 diabetes ' , Journal of Diabetes and its Complications, vol. 30 , no. 6 , pp. 1144-1150 . https://doi.org/10.1016/j.jdiacomp.2016.03.028

http://hdl.handle.net/10138/224125

https://doi.org/10.1016/j.jdiacomp.2016.03.028

publishedVersion

Downloaded from Helda, University of Helsinki institutional repository.

This is an electronic reprint of the original article.

This reprint may differ from the original in pagination and typographic detail.

Please cite the original version. 


\title{
Dietary patterns are associated with various vascular health markers and complications in type 1 diabetes
}

\author{
Aila J. Ahola a,b,c ${ }^{\text {, Riitta Freese }}{ }^{\mathrm{d}}$, Sari Mäkimattila ${ }^{\mathrm{a}}$, Carol Forsblom ${ }^{\mathrm{a}, \mathrm{b}, \mathrm{c}}$,

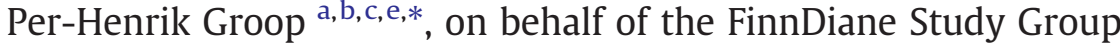 \\ ${ }^{a}$ Folkhälsan Institute of Genetics, Folkhälsan Research Center, Helsinki, Finland \\ b Abdominal Center Nephrology, University of Helsinki and Helsinki University Central Hospital, Helsinki, Finland

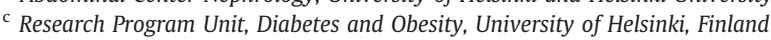 \\ d Division of Nutrition, Department of Food and Environmental Sciences, University of Helsinki, Finland \\ e Baker IDI Heart E' Diabetes Institute, Melbourne, Australia
}

\section{A R T I C L E I N F O}

\section{Article history:}

Received 29 February 2016

Received in revised form 24 March 2016

Accepted 25 March 2016

Available online 31 March 2016

\section{Keywords:}

Complications

Dietary pattern

Dietary recommendations

Type 1 diabetes

Vascular health markers

\begin{abstract}
A B S T R A C T
Aims: Diet plays an important role in the management of type 1 diabetes. However, the association between dietary intake and health has not been extensively studied in this population. We studied the cross-sectional association between dietary factors, and selected vascular health markers and complications in type 1 diabetes.

Methods: Data from 874 individuals with type 1 diabetes participating in the FinnDiane Study were included. Dietary intake was assessed using a self-reported questionnaire and a diet score, expressing the extent to which individuals adhered to the dietary recommendations, was calculated. Diet questionnaire was also used to reveal dietary patterns using factor analysis.

Results: Seven factors with high degree of inter-correlation were formed; healthy, traditional, vegetable, sweets, modern, low-fat cheese, and fish and eggs. In multivariate models, higher diet score and healthy factor score were associated with better glycaemic control. Higher diet score was associated with higher, while sweets, and fish and eggs patterns were associated with lower systolic blood pressure. Healthy, sweets, and fish and eggs factors were additionally associated with lower diastolic blood pressure.

Conclusions: Closer adherence to the dietary recommendations, and a diet high in fresh vegetables, fruits and berries, cooked vegetables, fish dishes, and yoghurt may be beneficial for the glycaemic control in type 1 diabetes. Moreover, a diet pattern with fish and eggs may have beneficial effects for blood pressure.
\end{abstract}

(C) 2016 Published by Elsevier Inc.

\section{Introduction}

Nutrition plays an integral role in the management of many chronic diseases, including type 1 diabetes. Among individuals with diabetes dietary recommendations aim, in particular, at reducing the risks of various long term complications. This is achieved by adapting dietary practices that, amongst others, support good glycaemic control, weight management, and appropriate intake of macro- and micronutrients. Dietary recommendations, targeted at individuals with diabetes, encourage the consumption of fruits and vegetables,

Conflicts of Interest: PHG has received research grants from Eli Lilly and Roche, is an advisory board member for AbbVie, Astra Zeneca, Boehringer-Ingelheim, Cebix, Eli Lilly, Janssen, MSD, Medscape, and Novartis. He has received lecture fees from Astra Zeneca, Boehringer-Ingelheim, Eli Lilly, Genzyme, Novartis, Novo Nordisk, Sanofi, and MSD. SM is an employee of Novo Nordisk A/S. All other authors declare that there is no conflict of interest.

* Corresponding author at: Folkhälsan Research Center, Biomedicum Helsinki C318b, PO Box 63, 00014, University of Helsinki, Finland. Tel.: + 3582941 25459; fax: + 3589 19125452.

E-mail address: per-henrik.groop@helsinki.fi (P-H. Groop). whole grain products, lean meats, fish, low fat liquid milk products, and vegetable oils, while the intakes of sugar, salt, and alcohol should be limited (Finnish Diabetes Association, 2008).

We have previously shown in the FinnDiane Study population that adherence to the dietary recommendations among individuals with type 1 diabetes is, in many aspects, suboptimal (Ahola, Mikkilä, Mäkimattila, et al., 2012). In particular, the intakes of carbohydrates and fiber are frequently lower than recommended, while the recommendations for salt, total fat, and saturated fat are often exceeded. Similar trends are seen in other studies of participants with type 1 diabetes (Leroux et al., 2014). While a number of studies have shown some health benefits of adhering to the dietary guidelines, most of them have been conducted in populations other than type 1 diabetes. For example, among middle-aged individuals with type 2 diabetes, closer adherence to dietary recommendations was associated with reduced risk of chronic kidney disease and death during a 5.5 year follow-up period (Dunkler et al., 2015), and in a population-based survey in the general population, better adherence to dietary guidelines was associated with lower fasting blood glucose 
concentrations, lower systolic blood pressure, and lower triglyceride concentration (Lee et al., 2013).

Some studies, conducted among individuals with type 1 diabetes, have assessed the role of selected dietary macro- and micronutrients on various health markers. In one such study, the percentage of energy from fats, saturated fats, and monounsaturated fats were all positively correlated with total and LDL cholesterol concentration, $\mathrm{HbA}_{1 \mathrm{c}}$, BMI, waist circumference, and diastolic blood pressure, and negatively with estimated glucose disposal rate (eGDR) (SnellBergeon et al., 2009). In the same study, increased carbohydrate intake was negatively correlated with total, LDL, and HDL cholesterol concentration, $\mathrm{HbA}_{1 \mathrm{c}}$, BMI, waist circumference, and diastolic blood pressure, but positively with eGDR. In another study, higher carbohydrate intake was associated with lower BMI and waist circumference, while higher saturated fat intake and lower intake of cereal fiber predicted higher waist-to-hip ratio (Toeller et al., 2001). Finally, lower intake of dietary fiber, polyunsaturated fatty acids, and vegetable protein, and higher intake of cholesterol were associated with endothelial dysfunction and low-grade inflammation (van Bussel et al., 2013).

Rather than focusing on individual macro- or micronutrients, however, there has been an increasing interest in the health consequences of various dietary patterns. It has been argued that a reductionist approach that focuses on isolated nutrients may oversimplify the complexity related to dietary intake at large (Sievenpiper \& Dworatzek, 2013). In general, there are two distinct approaches related to dietary patterns. In a hypothesis-orientated approach, dietary patterns are constructed based on available scientific evidence about the health effects of given dietary variables. In an exploratory approach, on the other hand, statistical methods are applied to the collected data in order to identify patterns, or clusters of dietary variables, that are inter-correlated.

The Dietary Approaches to Stop Hypertension (DASH) dietary pattern, an example of the hypothesis-orientated approach, that emphasizes the intake of fruits, vegetables, low-fat dairy, whole grains, fish, nuts (US Department of Health and Human Services, National Institutes of Health, National Heart, Lung, and Blood Institute, 1998) was, among the youth with type 1 diabetes, inversely associated with the LDL-HDL cholesterol ratio, and $\mathrm{HbA}_{1 \mathrm{c}}$ (Liese et al., 2011). On the other hand, adherence to a carbohydrateconscious Mediterranean diet pattern, compared to a low-fat diet, was associated with improved glycaemic control in newly diagnosed individuals with type 2 diabetes (Esposito et al., 2009). Improvements in the cardiovascular risk factor profile, with adherence to the Mediterranean diet, were also reported in moderately obese subjects with or without type 2 diabetes (Shai et al., 2008).

Our aim was therefore to study the association between adherence to the dietary recommendations and diet patterns, derived from exploratory analyses, and selected vascular health markers in adults with type 1 diabetes. We also studied the association between dietary intake and complications. These cross-sectional analyses serve as a baseline for future prospective analyses in this population.

\section{Subjects, materials and methods}

\subsection{Study subjects}

A total of 874 participants in the Finnish Diabetic Nephropathy (FinnDiane) Study were included in these cross-sectional analyses. Included were all individuals with type 1 diabetes who had filled in the diet questionnaire within 2 years of the study visit, and whose renal status and insulin dose were known. The Ethics Committee of the Hospital District of Helsinki and Uusimaa approved the study protocol. Signed informed consent was obtained from all participants prior to study inclusion.
2.2. Anthropometric measurements, blood pressure, blood samples, and smoking

Participants' height and weight were measured in light clothing, and body mass index $\left(\mathrm{kg} / \mathrm{m}^{2}\right)$ was calculated. Waist-to-hip ratio was calculated from the respective measurements. Following a 10-minute rest, blood pressure was measured in the sitting position. A second blood pressure measurement was conducted after a further 2 minute rest. The means of these two measurements were used in the analyses. Blood samples were collected and $\mathrm{HbA}_{1 \mathrm{c}}$ was determined locally using standardised assays. Serum lipid and lipoprotein concentrations were measured as previously described (Thorn et al., 2005). Data on smoking and insulin dose were self-reported.

\subsection{Urinary albumin excretion rate and complications}

Urinary albumin excretion rate (AER) in at least two out of three timed 24-hour or overnight urine collections was used to assess participants' renal status. Participants were classified according to the AER: normal albumin excretion rate (AER $<20 \mu \mathrm{g} / \mathrm{min}$ or $<30 \mathrm{mg} /$ $24 \mathrm{~h}$ ), microalbuminuria (AER $\geq 20$ and $<200 \mu \mathrm{g} / \mathrm{min}$ or $\geq 30$ and $<300 \mathrm{mg} / 24 \mathrm{~h}$ ), macroalbuminuria (AER $\geq 200 \mu \mathrm{g} / \mathrm{min}$ or $\geq 300 \mathrm{mg} /$ $24 \mathrm{~h}$ ), and end-stage renal disease (ESRD) (in dialysis or with kidney transplant). Advanced chronic kidney disease (CKD) was defined as macroalbuminuria or ESRD. Data on retinopathy and cardiovascular events were obtained from the medical records. Retinal laser-treatment was used as indication of severe retinopathy. Acute myocardial infarction, coronary bypass, stroke, amputation, and peripheral vascular disease were grouped as "major cardiovascular events".

\subsection{Diet}

The diet questionnaire, that was used to collect the data on dietary intake, was previously described in detail (Ahola, Mikkilä, Mäkimattila, et al., 2012). In short, the self-reported questionnaire was designed to capture information on the participants' habitual dietary intake, and included questions regarding the consumption pattern of food items such as coffee, milk products, breads, spreads, cooking fats, and salt. As part of the form, a 19-item food frequency questionnaire (FFQ) was also included. In the FFQ the consumption frequencies of the most common food items in Finland were queried. On a seven point response scale, ranging from "multiple times per day" to "once a month or less frequently", participants reported their intakes of fish dishes, meat dishes, poultry, sausages and cold cuts, eggs, legumes, fresh vegetables, cooked vegetables, potatoes, pasta and rice, fruits and berries, high-fat cheese, low-fat cheese, yoghurt, ice cream, soft drinks, pastries, candy, and fried and grilled foods. Based on the replies on the diet questionnaire, a diet score was calculated for each participant, as previously described (Ahola, Mikkilä, Saraheimo, et al., 2012). The principals for calculating the score are also presented in the supplementary Table 2 . Scores, ranging between 0 and 22 points, express the extent to which participants comply with the dietary recommendations. The higher the score, the better the adherence. The diet score is used as a continuous variable in the analyses. The FFQ part of the questionnaire was analysed by exploratory factor analysis, as described in the statistical analyses, and the obtained factor scores were used in further analyses.

\subsection{Leisure time physical activity}

Participants' leisure time physical activity (LTPA) was evaluated with a questionnaire (Wadén et al., 2005) in which participants retrospectively reported mean frequency, single session duration, and intensity of 21 common forms of physical activity for the past 12 months. The amount of weekly physical activity, displayed as metabolic equivalent of task hour (METh), was calculated by multiplying the reported duration of the activity by the activity- and intensity-specific metabolic equivalent. 


\subsection{Statistical analyses}

Descriptive statistics were reported as percentages for categorical variables, mean \pm standard deviation (SD) for normally distributed continuous variables, and median (interquartile range) for nonnormally distributed continuous variables. The respective group comparisons were performed with chi-squared test, independentsample t-test, and Mann-Whitney U-test. Exploratory factor analysis (maximal likelihood and varimax rotation) was used to identify underlying dietary constructs within the FFQ part of the diet questionnaire. In the analysis, the number of factors identified was based on eigenvalues $>1.0$, and items with factor loading $|\geq 0.20|$ with a particular factor, were included. The factor score was the sum of the scores for all items associated with that particular factor multiplied by its corresponding factor loading. Correlations between calculated diet score and the obtained factor scores were investigated with Spearman correlation coefficients. Moreover, the factor scores were used as independent variables in the subsequent analyses. Generalised linear model was used to investigate the independent associations between dietary variables and a number of outcome variables (waist-to-hip ratio, $\mathrm{HbA}_{1 \mathrm{c}}$, systolic blood pressure, diastolic blood pressure, and LDL cholesterol and triglyceride concentrations). Analysis of covariance was used to study the association between diet and factor scores, and complication status. For these analyses, significant variables from the bivariate analyses were selected as covariates. Bonferroni correction, for multiple testing, was applied for these results. All analyses were conducted using IBM SPSS Statistics for Windows, Version 22.0 (IBM Corp, Armonk, NY, USA).

\section{Results}

Data from a total of 874 individuals ( $45 \%$ men) were included in the analyses. Mean \pm SD age of the population was $45.8 \pm 13.1$ years. Patient characteristics, divided by gender, are shown in Table 1 . Compared to men, the median diet score was higher among women [11 (8-13) vs. 12 (10-15), $p<0.001]$.

The factor analysis yielded seven dietary factors with high degree of inter-correlation. The factors were named healthy, traditional, vegetable, sweets, modern, low-fat cheese, and fish and eggs, based on the food items included in each factor (Table 2). Factor loadings for the dietary items included in each factor are shown in the supplementary Table 1 . Women got higher scores in the healthy factor, while men scored higher in the traditional and vegetable factors (Table 3 ).

The diet score was positively correlated with the healthy factor score $(r=0.633, p<0.001)$, the vegetable factor score $(r=0.127$, $p<0.001)$, the low-fat cheese factor score $(r=0.144, p<0.001)$, and

Table 1

Participant characteristics divided by gender.

\begin{tabular}{lllr}
\hline & $\begin{array}{l}\text { Menn }=393 \\
(45 \%)\end{array}$ & $\begin{array}{l}\text { Womenn }=481 \\
(55 \%)\end{array}$ & $p$ \\
\hline Age, years & $47.2(36.9-56.7)$ & $45.2(34.8-54.5)$ & 0.031 \\
Current smoking, \% & 13.2 & 13.5 & 0.919 \\
Advanced CKD, \% & 23.9 & 16.2 & 0.005 \\
BMI, kg/m $^{2}$ & $25.6(23.6-27.8)$ & $24.9(22.6-28.0)$ & 0.014 \\
HbA ${ }_{1 c}$ \% & $8.1(7.4-8.7)$ & $8.1(7.4-9.1)$ & 0.137 \\
Total cholesterol, mmol/l & $4.4(3.9-4.8)$ & $4.6(4.1-5.2)$ & $<0.001$ \\
HDL cholesterol, mmol/l & $1.43(1.15-1.69)$ & $1.71(1.44-2.05)$ & $<0.001$ \\
Triglycerides, mmol/l & $1.02(0.79-1.41)$ & $0.87(0.65-1.19)$ & $<0.001$ \\
Systolic blood pressure, mmHg & $140(131-153)$ & $133(121-146)$ & $<0.001$ \\
Diastolic blood pressure, mmHg & $78(72-85)$ & $76(70-83)$ & 0.022 \\
Insulin dose, IU/kg & $0.63(0.48-0.79)$ & $0.59(0.47-0.75)$ & 0.026 \\
Diet score & $11(8-13)$ & $12(10-15)$ & $<0.001$ \\
METh & $19.1(7.6-32.8)$ & $15.8(7.2-29.5)$ & 0.336 \\
\hline
\end{tabular}

Data are presented as median (interquartile range) or frequencies (\%).CKD, chronic kidney disease; BMI, body mass index; METh, metabolic equivalent of task hours.
Table 2

Formed dietary factors.

\begin{tabular}{|c|c|c|c|}
\hline Factor & Included items & Eigenvalue & $\begin{array}{l}\% \text { of } \\
\text { variance }\end{array}$ \\
\hline Healthy & $\begin{array}{l}\text { Fresh vegetables, fruits and berries, } \\
\text { cooked vegetables, fish dishes, yoghurt }\end{array}$ & 2.19 & 11.55 \\
\hline Traditional & $\begin{array}{l}\text { Meat dishes, potatoes, sausages and } \\
\text { cold cuts }\end{array}$ & 1.91 & 10.08 \\
\hline Vegetable & Legumes, cooked vegetables & 1.57 & 8.29 \\
\hline Sweets & Candies, pastries, ice cream, yoghurt & 1.42 & 7.48 \\
\hline Modern & $\begin{array}{l}\text { Pasta and rice, poultry, fried foods, } \\
\text { meat dishes }\end{array}$ & 1.34 & 7.05 \\
\hline $\begin{array}{l}\text { Low-fat } \\
\text { cheese }\end{array}$ & Low-fat cheese, low in high-fat cheese & 1.13 & 5.92 \\
\hline Fish and eggs & Eggs, fish dishes & 1.04 & 5.02 \\
\hline
\end{tabular}

the fish and eggs factor score $(r=0.090, p=0.013)$. Moreover, the diet score was negatively correlated with the sweets factor score $(r=-0.433, p<0.001)$, and showed no correlation with the modern factor score $(r=0.064, p=076)$.

In a multivariate analysis, adjusted for gender, age, advanced CKD, smoking, insulin dose, and physical activity, higher diet scores were associated with lower $\mathrm{HbA}_{1 \mathrm{c}}$ (Table 4). After further adjustment for the use of antihypertensive medication, higher diet scores were associated with higher systolic blood pressure. Higher scores in the healthy factor were associated with better glycaemic control and lower diastolic blood pressure. Higher scores in the traditional factor were associated with higher waist-to-hip ratio. Higher scores in the sweets factor were associated with lower systolic and diastolic blood pressure. Similarly, higher scores in the fish and eggs factor were associated both with lower systolic and diastolic blood pressure.

In separate analyses among men, higher scores in the healthy factor were associated with lower diastolic blood pressure. Higher scores in the sweets factor were associated with lower systolic blood pressure, and lower LDL cholesterol concentration. Finally, higher scores in the low-fat cheese were associated with lower triglyceride concentrations. Among women, higher diet scores were associated with higher systolic blood pressure. Higher scores in the healthy factor were associated both with lower waist-to-hip ratio and better glycaemic control. Higher scores in the vegetable factor were associated with higher systolic blood pressure, while higher scores in the fish and eggs factor were associated both with lower systolic and diastolic blood pressures.

A total of 172 (19.7\%) individuals had advanced CKD (Table 5). Data on retinopathy status was available from 868 participants. Of these individuals 302 (34.8\%) had severe retinopathy. Data on major cardiovascular event status were available from 871 participants, of which 128 (14.7\%) had experienced at least one of the previously listed cardiovascular events. In the multivariate models, having advanced CKD was associated $(p<0.05)$ with lower scores in the sweets and low-fat cheese factors (Table 6 ), and having retinopathy was associated with higher diet score, higher healthy factor score, and lower low-fat cheese factor score. However, of the above mentioned observations, after Bonferroni correction, the only significant $(p<0.002)$ finding was the association

Table 3

Dietary factor scores divided by gender.

\begin{tabular}{llrr}
\hline Factor & Men & Women & \multicolumn{1}{l}{$p$} \\
\hline Healthy & $-0.40(-0.80$ to 0.21$)$ & $0.20(-0.41$ to 0.85$)$ & $<0.001$ \\
Traditional & $-0.15(-0.39$ to 0.75$)$ & $-0.27(-0.76$ to 0.45$)$ & $<0.001$ \\
Vegetable & $-0.24(-0.49$ to 0.09$)$ & $-0.37(-0.62$ to 0.03$)$ & $<0.001$ \\
Sweets & $-0.19(-0.58$ to 0.32$)$ & $-0.16(-0.51$ to 0.42$)$ & 0.298 \\
Modern & $-0.14(-0.53$ to 0.33$)$ & $-0.08(-0.49$ to 0.44$)$ & 0.163 \\
Low-fat cheese & $-0.05(-0.50$ to 0.43$)$ & $-0.02(-0.39$ to 0.44$)$ & 0.197 \\
Fish and eggs & $-0.10(-0.36$ to 0.25$)$ & $-0.16(-0.43$ to 0.23$)$ & 0.067 \\
\hline
\end{tabular}

Data are presented as median (interquartile range). 
between the presence of retinopathy and lower low-fat cheese score. Having experienced any major cardiovascular event was not independently associated with any of the studied dietary variables.

\section{Discussion}

In the current cross-sectional study among individuals with type 1 diabetes, a number of associations between diet and selected vascular health markers and complications were observed. Important to individuals with diabetes, a diet that more closely adhered to the dietary recommendations was associated with better glycaemic control. Lower $\mathrm{HbA}_{1 \mathrm{c}}$ levels were also related to a dietary factor named healthy, which was observed to highly correlate with the diet score. Healthy, sweet, and fish and eggs factors were all associated with lower blood pressure measurements, while a diet that more closely adhered to the dietary recommendations was also associated with higher systolic blood pressure.

Individuals with advanced CKD and retinopathy showed a tendency towards healthier diets, as advanced CKD was independently associated with lower scores in the sweet factor, and retinopathy with higher diet scores and healthy factor scores. In the bivariate analyses, major cardiovascular events were associated with higher diet scores, and lower scores in the sweets and modern factors. After adjustments with age, diabetes duration, dyslipidaemia, and blood pressure, however, having experienced some major cardiovascular event was not independently associated with any dietary factor, observed. Moreover, after Bonferroni correction for multiple testing, none of the above observations remained significant.

The calculation of the diet score was based on the extent to which individuals adhere to the dietary recommendations, reflecting the current level of scientific evidence, and may thus be seen as a hypothesis-orientated approach of looking at dietary patterns. Using factors derived from factor analysis, on the other hand, represent an exploratory approach. Of the formed factors, the healthy factor was observed to be most strongly positively correlated with the calculated diet score. Indeed, vegetables, fruits and berries, as well as fish, included in the healthy factor, are also included in the calculation of the diet score. A number of differences between these, apparently similar sounding scores exist, however, as low-fat liquid milk products, vegetable based fats, and salt were not accounted for in the healthy factor. These differences may explain some of the differences observed in the results between the two approaches. Candy, pastries, and ice cream, found both in the sweets factor and the diet score, on the other hand, explain the significant negative correlation observed between these variables.

Due to a limited number of studies related to dietary patterns, and cardiovascular risk factors and complications, conducted among individuals with type 1 diabetes, it is difficult to directly compare our findings to previous research. Some studies are, however, sufficiently comparable. One such study explored the association between cardiovascular risk factors and the DASH diet pattern, a pattern that fairly closely resembles the healthy factor or the diet score, referred to in our study (US Department of Health and Human Services, National Institutes of Health, National Heart, Lung, and Blood Institute, 1998). Similar to our observations, Liese et al. (2011) observed, amongst youth with type 1 diabetes, that adherence to the DASH diet was inversely associated with $\mathrm{HbA}_{1 \mathrm{c}}$.

Another dietary pattern frequently investigated is the so called Mediterranean dietary pattern with abundant use of fruits, vegetables, legumes, nuts, fish, and olive oil. The Mediterranean diet also shares many similarities with the healthy factor and the diet score used in the current study. Related to the adherence to the Mediterranean diet in type 2 diabetes, Esposito et al. (2009) observed benefits regarding glycaemic control. In a cross-sectional study of 8,821 adults, closer adherence to the Mediterranean diet pattern was associated with lower likelihood of central adiposity, high triglyceride levels, and hypertension (Grosso et al., 2015). Moreover, a systematic review, investigating the association between the Mediterranean dietary pattern and diabetes control and cardiovascular risk modification, concluded that most included studies showed favourable effects of adherence to the diet and glycaemic control and cardiovascular disease, while some degree of controversy remains with respect to obesity (Sleiman, Al-Badri, \& Azar, 2015).

In individuals with type 1 diabetes, adherence to the Mediterranean diet has been associated with lower trunk fat percentage, BMI, waist circumference, and systolic and diastolic blood pressure (Gingras et al., 2015). In the same study, a diet that met at least three of the six dietary recommendations related to carbohydrate, protein, fat, saturated fat, fibre, and sodium intake, was only associated with lower trunk fat percentage. In another study including youth with type 1 diabetes, healthy eating index was not associated with $\mathrm{HbA}_{1 \mathrm{c}}$ values (Nansel, Haynie, Lipsky, Laffel, \& Mehta, 2012). Greater number of daily fruit servings and higher whole-grain intake were, however, associated with lower BMI percentile. In a population of 162 healthy middle-aged individuals, adherence to dietary guidelines for a duration of 12 weeks, as opposed to control traditional British diet, was associated with reduction in blood pressure and improved lipid profile (Reidlinger et al., 2015). Finally, in a cross-sectional study among women with type 2 diabetes, fish and shellfish intake was negatively associated with triglyceride concentration and pulse wave velocity (Kim et al., 2015).

Among the studies identifying dietary factors using food frequency questionnaire and factor analysis, similar to us, is a Chinese study of 2,900 participants aged 30 years or older (Hong, Xu, Wang, Liang, \& Li, 2015). In that study, a healthy traditional diet pattern, rich in green vegetables, rice, vegetables, and fish and shrimp, was associated with lower risk of hyperglycaemia after a three year follow-up. On the other hand, a diet factor with fruits, eggs, and juices was associated with increased hyperglycaemia risk.

A number of prospective cohort, cross-sectional, and clinical studies have been conducted to investigate the association between dietary patterns and complications. With respect to such studies, a recent review of 10 studies concluded that adherence to the Mediterranean diet pattern was associated with a significant reduction in coronary vascular diseases (Garcia-Fernandez, RicoCabanas, Rosgaard, Estruch, \& Bach-Faig, 2014). In a cross-sectional study, higher intake of fish and vegetables was associated with lower creatinine levels and marginally higher eGFR levels in a population of individuals with type 2 diabetes (Hsu et al., 2014). In the Nurses' Health Study, a western dietary pattern was associated with increased odds of microalbuminuria and rapid kidney function decrease, while a DASH dietary pattern showed no association with microalbuminuria but was associated with decreased risk of rapid eGFR decline (Lin, Fung, Hu, \& Curhan, 2011). In the same study, a prudent dietary pattern was not associated with either microalbuminuria or eGFR decline. Finally, during a 5.5 year follow-up, adherence to the dietary recommendations was associated with lower risk of kidney disease in a European population of individuals with type 2 diabetes (Dunkler et al., 2015).

A large number of well characterised participants provide sufficient power to study the associations between dietary intake, and vascular health markers and complications. There are, however, a number of limitations related to our study. First, self-reported dietary assessments are prone to error. This is true in particular with respect to studies assessing energy intake, as seen in the National Health and Nutrition Examination Survey, where 67\% of women and 59\% of men reported physiologically implausible intakes (Archer, Hand, \& Blair, 2013). Instead of assessing energy intake, however, we aimed at estimating the overall quality of the diet using a diet questionnaire, which has its own specific limitations. Questionnaires not only rely on memory, but some individuals may also be prone to give responses that are considered socially acceptable. The latter typically leads to underreporting the unhealthy and over-reporting the healthy habits. 
Table 4

Associations between diet score, dietary factors and selected health outcomes.

\begin{tabular}{|c|c|c|c|c|c|c|}
\hline & WHR & $\mathrm{HbA}_{1 \mathrm{c}}$ & SBP & DBP & LDL & TG \\
\hline \multicolumn{7}{|l|}{ Diet score } \\
\hline All & $-0.001(-0.003$ to 0.001$)$ & $-0.032(-0.059$ to -0.004$)$ & $0.523(0.170$ to 0.876$)$ & $-0.014(-0.229$ to 0.201$)$ & $0.014(-0.014$ to 0.043$)$ & $-0.013(-0.029$ to 0.002$)$ \\
\hline Men & $-0.002(-0.004$ to 0.001$)$ & $-0.034(-0.069$ to 0.001$)$ & $0.389(-0.113$ to 0.891$)$ & $-0.049(-0.373$ to 0.275$)$ & $0.034(-0.005$ to 0.074$)$ & $-0.015(-0.037$ to 0.007$)$ \\
\hline Women & $-0.002(-0.004$ to 0.000$)$ & $-0.032(-0.074$ to 0.009$)$ & 0.644 (0.150 to 1.138$)$ & $-0.001(-0.290$ to 0.288$)$ & $-0.014(-0.054$ to 0.026$)$ & $-0.012(-0.035$ to 0.011$)$ \\
\hline \multicolumn{7}{|l|}{ Healthy } \\
\hline All & -0.007 ( -0.016 to 0.001$)$ & $-0.183(-0.323$ to -0.042$)$ & 0.517 ( -1.347 to 2.381$)$ & $-1.203(-2.310$ to -0.096$)$ & -0.016 ( -0.164 to 0.132$)$ & $-0.019(-0.103$ to 0.065$)$ \\
\hline Men & $-0.005(-0.020$ to 0.011$)$ & $-0.146(-0.346$ to 0.054$)$ & $-1.682(-4.724$ to 1.361$)$ & $-2.576(-4.456$ to -0.696$)$ & $-0.057(-0.301$ to 0.187$)$ & $-0.076(-0.209$ to 0.058$)$ \\
\hline Women & $-0.009(-0.018$ to -0.001$)$ & $-0.197(-0.390$ to -0.004$)$ & $1.804(-0.536$ to 4.144$)$ & -0.385 ( -1.744 to 0.973$)$ & $-0.030(-0.212$ to 0.153$)$ & $0.017(-0.093$ to 0.127$)$ \\
\hline \multicolumn{7}{|l|}{ Traditional } \\
\hline All & 0.007 (0.001 to 0.013 ) & $0.099(-0.004$ to 0.202$)$ & -0.499 ( -1.876 to 0.878$)$ & $0.152(-0.670$ to 0.973$)$ & $0.036(-0.103$ to 0.314$)$ & -0.007 ( -0.067 to 0.052$)$ \\
\hline Men & $0.009(-0.001$ to 0.020$)$ & $0.035(-0.102$ to 0.171$)$ & -0.047 ( -2.118 to 2.025$)$ & $0.088(-1.210$ to 1.387$)$ & -0.059 ( -0.197 to 0.079$)$ & $0.083(-0.002$ to 0.168$)$ \\
\hline Women & $0.006(-0.001$ to 0.013$)$ & $0.134(-0.014$ to 0.283$)$ & $-1.010(-2.837$ to 0.816$)$ & $0.181(-0.878$ to 1.240$)$ & $0.107(-0.022$ to 0.235$)$ & $-0.073(-0.156$ to 0.009$)$ \\
\hline \multicolumn{7}{|l|}{ Vegetable } \\
\hline All & $-0.002(-0.008$ to 0.004$)$ & $-0.032(-0.132$ to 0.067$)$ & $0.262(-1.051$ to 1.575$)$ & 0.090 ( -0.693 to 0.874$)$ & $0.104(-0.019$ to 0.228$)$ & $0.037(-0.019$ to 0.094$)$ \\
\hline Men & $-0.003(-0.011$ to 0.006$)$ & $-0.097(-0.209$ to 0.014$)$ & -0.905 ( -2.604 to 0.794$)$ & $-0.346(-1.413$ to 0.720$)$ & -0.005 ( -0.196 to 0.185$)$ & 0.045 ( -0.023 to 0.113$)$ \\
\hline Women & $-0.002(-0.010$ to 0.005$)$ & $0.059(-0.114$ to 0.232$)$ & 2.079 (0.032 to 4.126$)$ & $0.788(-0.402$ to 1.977$)$ & $0.154(-0.016$ to 0.323$)$ & $0.033(-0.065$ to 0.131$)$ \\
\hline \multicolumn{7}{|l|}{ Sweets } \\
\hline All & $-0.002(-0.010$ to 0.006$)$ & $0.069(-0.065$ to 0.202$)$ & $-2.618(-4.395$ to -0.842$)$ & $-1.168(-2.232$ to -0.104$)$ & $-0.007(-0.113$ to 0.300$)$ & 0.025 ( -0.052 to 0.102$)$ \\
\hline Men & $-0.003(-0.017$ to 0.010$)$ & $0.042(-0.139$ to 0.222$)$ & $-3.794(-6.522$ to -1.065$)$ & $-1.454(-3.183$ to 0.275$)$ & $-0.282(-0.498$ to -0.065$)$ & $-0.019(-0.135$ to 0.097$)$ \\
\hline Women & $-0.003(-0.011$ to 0.006$)$ & $0.092(-0.097$ to 0.280$)$ & $-1.870(-4.175$ to 0.434$)$ & $-1.027(-2.361$ to 0.307$)$ & $0.123(-0.029$ to 0.275$)$ & $0.053(-0.051$ to 0.157$)$ \\
\hline \multicolumn{7}{|l|}{ Modern } \\
\hline All & $0.003(-0.006$ to 0.011$)$ & $0.051(-0.095$ to 0.198$)$ & $-0.140(-2.071$ to 1.790$)$ & $-0.012(-1.163$ to 1.140$)$ & $0.050(-0.099$ to 0.199$)$ & $-0.028(-0.116$ to 0.061$)$ \\
\hline Men & $0.005(-0.010$ to 0.020$)$ & $-0.059(-0.258$ to 0.140$)$ & $1.133(-1.819$ to 4.085$)$ & $0.238(-1.615$ to 2.090$)$ & $0.092(-0.133$ to 0.317$)$ & $0.006(-0.130$ to 0.143$)$ \\
\hline Women & $0.001(-0.009$ to 0.010$)$ & $0.132(-0.077$ to 0.341$)$ & $-1.035(-3.569$ to 1.499$)$ & $-0.111(-1.579$ to 1.357$)$ & $0.077(-0.117$ to 0.271$)$ & $-0.043(-0.160$ to 0.074$)$ \\
\hline \multicolumn{7}{|c|}{ Low-fat cheese } \\
\hline All & $-0.008(-0.017$ to 0.001$)$ & $-0.112(-0.260$ to 0.035$)$ & $1.700(-0.270$ to 3.669$)$ & $0.302(-0.876$ to 1.480$)$ & $-0.067(-0.217$ to 0.083$)$ & $-0.082(-0.168$ to 0.004$)$ \\
\hline Men & $-0.012(-0.027$ to 0.003$)$ & $-0.139(-0.337$ to 0.059$)$ & $0.839(-2.194$ to 3.873$)$ & $-0.154(-2.057$ to 1.748$)$ & -0.220 ( -0.447 to 0.006$)$ & $-0.188(-0.313$ to -0.063$)$ \\
\hline Women & $-0.005(-0.015$ to 0.005$)$ & $-0.094(-0.303$ to 0.116$)$ & $2.210(-0.346$ to 4.767$)$ & $0.587(-0.898$ to 2.072$)$ & $0.020(-0.172$ to 0.212$)$ & $-0.009(-0.126$ to 0.107$)$ \\
\hline \multicolumn{7}{|c|}{ Fish and eggs } \\
\hline All & -0.004 ( -0.013 to 0.006$)$ & $-0.082(-0.258$ to 0.094$)$ & $-3.426(-5.573$ to -1.279$)$ & $-1.346(-2.634$ to -0.058$)$ & $0.001(-0.218$ to 0.221$)$ & -0.016 ( -0.122 to 0.090$)$ \\
\hline Men & $-0.006(-0.020$ to 0.009$)$ & $-0.139(-0.357$ to 0.079$)$ & $-2.854(-5.709$ to 0.001$)$ & $-0.373(-2.178$ to 1.432$)$ & $-0.114(-0.394$ to 0.167$)$ & $-0.005(-0.147$ to 0.138$)$ \\
\hline Women & $-0.007(-0.020$ to 0.005$)$ & $-0.038(-0.309$ to 0.233$)$ & $-4.155(-7.402$ to -0.908$)$ & $-2.676(-4.550$ to -0.802$)$ & $0.108(-0.222$ to 0.439$)$ & $-0.014(-0.172$ to 0.145$)$ \\
\hline
\end{tabular}

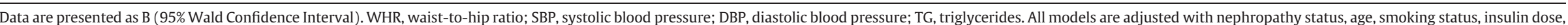

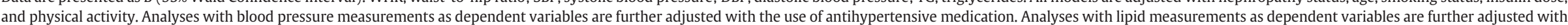
the use of lipid lowering medication. Joint analyses of men and women are further adjusted with gender. Generalised linear model. 
Table 5

Diet scores and factor scores divided by complication status.

\begin{tabular}{|c|c|c|c|c|c|c|}
\hline & \multicolumn{2}{|c|}{ Advanced chronic kidney disease } & \multicolumn{2}{|l|}{ Retinopathy } & \multicolumn{2}{|c|}{ Major cardiovascular event } \\
\hline & Non $=702(80.3 \%)$ & Yesn $=172(19.7 \%)$ & Non $=566(65.2 \%)$ & Yesn $=302(34.8 \%)$ & Non $=743(85.3 \%)$ & Yesn $=128(14.7 \%)$ \\
\hline Diet & $11(9-14)$ & $12(9-14)$ & $11(9-14)$ & $12(9-14)$ & $11(9-14)$ & $13(10-14)^{b}$ \\
\hline Healthy & $-0.09(-0.62$ to 0.61$)$ & $-0.18(-0.62$ to 0.58$)$ & $-0.11(-0.64$ to 0.62$)$ & $-0.09(-0.57$ to 0.58$)$ & $-0.11(-0$ & $-0.05 i$ \\
\hline Traditional & $-0.21(-0.63$ to 0.69$)$ & $-0.21(-0.63$ to 0.53$)$ & $-0.20(-0.63$ to 0.69$)$ & $-0.23(-0.63$ to 0.60$)$ & $-0.21(-0.63$ to 0.69$)$ & $-0.18(-0.61$ to 0.59$)$ \\
\hline Vegetable & $-0.31(-0.55$ to 0.09$)$ & $-0.27(-0.57$ to 0.04$)$ & -0.31 (0.53 to 0.09$)$ & $-0.28(-0.59$ to 0.05$)$ & $-0.31(-0.55$ to 0.10$)$ & $-0.32(-0.61$ to -0.03$)$ \\
\hline Sweets & $-0.12(-0.49$ to 0.43$)$ & $-0.35(-0.69 \text { to } 0.12)^{a}$ & $-0.09(-0.45$ to 0.44$)$ & $-0.33(-0.68 \text { to } 0.12)^{a}$ & $-0.12(-0.50$ to 0.40$)$ & $-0.43(-0.75 \text { to } 0.12)^{a}$ \\
\hline Modern & $-0.07(-0.45$ to 0.43$)$ & $-0.26(-0.70 \text { to } 0.30)^{b}$ & $-0.03(-0.42$ to 0.47$)$ & $-0.23(-0.64 \text { to } 0.31)^{a}$ & $-0.07(-0.45$ to 0.44$)$ & $-0.32(-0.74 \text { to } 0.13)^{a}$ \\
\hline Low-fat cheese & $0.02(-0.44$ to 0.50$)$ & $-0.18(-0.40 \text { to } 0.20)^{c}$ & $0.05(-0.42$ to 0.53$)$ & $-0.10(-0.46 \text { to } 0.21)^{b}$ & $-0.02(-0.44$ to 0.47$)$ & $-0.10(-0.36$ to 0.30$)$ \\
\hline Fish and eggs & $-0.13(-0.3$ to 0.23$)$ & $-0.17(-0.43$ to 0.22$)$ & $-0.14(-0.38$ to 0.19$)$ & $-0.15(-0.41$ to 0.29$)$ & $-0.15(-0.41$ to 0.22$)$ & $-0.11(-0.37$ to 0.32$)$ \\
\hline
\end{tabular}

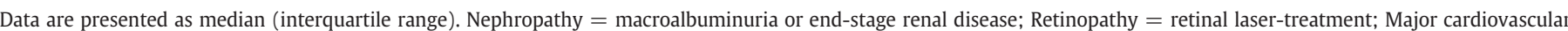

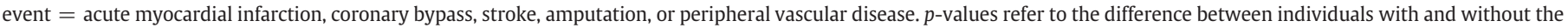
complication in question.

${ }^{\text {a }} p<0.001$.

b $p<0.01$.

c $p<0.05$.

Should this have taken place, it would have most likely diluted the results. The factor analysis used to form dietary factors, instead of using predetermined dietary patters, such as that of the Mediterranean dietary pattern, may not easily translate into practical recommendations. Dietary patterns may be more easily prescribed compared to specific food groups, identified in the current study. Frequent hypoglycaemias in individuals with type 1 diabetes, may affect the overall diet. However, in the current study, hypoglycaemia frequency was not taken into consideration. The current results need to be interpreted in the light of this limitation. Compared to those not participating, individuals taking part in health related studies may also be more health conscious. It is plausible that such selection bias has also taken place in the current study and has, again, likely diluted the observed effects. Another limitation is related to the cross-sectional nature of the study. This approach will not allow us to draw causal inferences from the results, but states only the observed associations. It is quite unlikely, for example, that high intake of candies, pastries, ice cream and yoghurt (the sweet factor) would be beneficial with respect to blood pressure. The more likely explanation is that individuals with diagnosed hypertension have made efforts to manage their blood pressure with healthier dietary choices. Emergence of complications, such as nephropathy and retinopathy, may similarly have shaped dietary intakes observed in the present study, and not the other way around. It is also possible that despite of adjusting for a number of variables, some residual confounding remains that could explain the observed association. The causalities will be studied in the future when prospective data will be available. Finally, due to multiple analyses, there is a possibility of false positive findings due to a chance. Thus, the current results must be interpreted in the light of this limitation.

In conclusion, the clinical message of the paper is that closer adherence to the dietary recommendations, and a diet abundant with fresh vegetables, fruits and berries, cooked vegetables, fish dishes, and yoghurt, may be beneficial for glycaemic control in type 1 diabetes. Moreover, a diet pattern with fish and eggs, on the other hand, may have beneficial effects for blood pressure.

\section{Funding}

This study was supported by grants from Academy of Finland, Novo Nordisk Foundation, Signe and Ane Gyllenberg Foundation, Folkhälsan Research Foundation, and Wilhelm and Else Stockmann Foundation.

\section{Acknowledgements}

The skilled technical assistance of Anna Sandelin, Jaana Tuomikangas, and Satu Kinnunen is gratefully acknowledged. The authors also acknowledge all the physicians and nurses at each centre participating in the collection of patients (online appendix).

\section{Appendix A. Supplementary data}

Supplementary data to this article can be found online at http://dx. doi.org/10.1016/j.jdiacomp.2016.03.028.

\section{References}

Ahola, A. J., Mikkilä, V., Mäkimattila, S., Forsblom, C., Freese, R., \& Groop, P. H. (2012a). Energy and nutrient intakes and adherence to dietary guidelines among Finnish adults with type 1 diabetes. Annals of Medicine, 44, 73-81.

Ahola, A. J., Mikkilä, V., Saraheimo, M., Wadén, J., Mäkimattila, S., \& Forsblom, C. (2012b). Sense of coherence, food selection and leisure time physical activity in type 1 diabetes. Scandinavian Journal of Public Health, 40, 621-628.

Table 6

The association between diet and factor scores and complications.

\begin{tabular}{|c|c|c|c|c|c|c|}
\hline & \multicolumn{2}{|l|}{ Advanced CKD } & \multicolumn{2}{|l|}{ Retinopathy } & \multicolumn{2}{|c|}{ Major cardiovascular event } \\
\hline & $F\left(d f_{\mathrm{M}}, d f_{\mathrm{R}}\right)$ & $p$ & $F\left(d f_{\mathrm{M}}, d f_{\mathrm{R}}\right)$ & $p$ & $F\left(d f_{\mathrm{M}}, d f_{\mathrm{R}}\right)$ & $p$ \\
\hline Diet score & $(1,732)=0.90$ & 0.343 & $(1,719)=8.26$ & 0.004 & $(1,731)=0.62$ & 0.432 \\
\hline Healthy & $(1,661)=0.57$ & 0.449 & $(1,650)=4.94$ & 0.027 & $(1,660)=2.02$ & 0.156 \\
\hline Traditional & $(1,661)=0.06$ & 0.814 & $(1,650)=1.71$ & 0.191 & $(1,660)=0.01$ & 0.925 \\
\hline Vegetable & $(1,661)=0.07$ & 0.788 & $(1,650)=0.05$ & 0.823 & $(1,660)=2.37$ & 0.124 \\
\hline Sweets & $(1,661)=4.89$ & 0.027 & $(1,650)=3.02$ & 0.083 & $(1,660)=2.37$ & 0.124 \\
\hline Modern & $(1,661)=0.54$ & 0.463 & $(1,650)=1.64$ & 0.201 & $(1,660)=0.07$ & 0.791 \\
\hline Low-fat cheese & $(1,661)=3.86$ & 0.049 & $(1,650)=13.63$ & $<0.001$ & $(1,660)=2.43$ & 0.120 \\
\hline Fish and eggs & $(1,661)=0.49$ & 0.484 & $(1,650)=0.15$ & 0.703 & $(1,660)=0.25$ & 0.620 \\
\hline
\end{tabular}

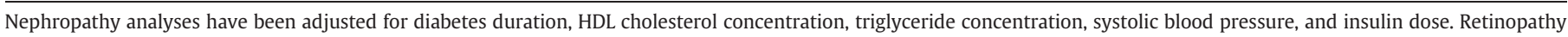

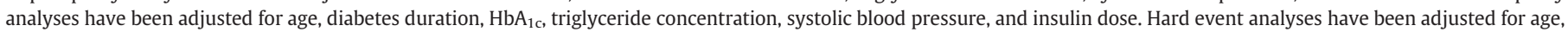
diabetes duration, HDL cholesterol concentration, systolic blood pressure, and diastolic blood pressure. CKD, chronic kidney disease. Analysis of covariance. 
Archer, E., Hand, G. A., \& Blair, S. N. (2013). Validity of U.S. nutritional surveillance: National Health and Nutrition Examination Survey caloric energy intake data, 1971-2010. PLOS ONE, 8, e76632.

Dunkler, D., Kohl, M., Teo, K. K., Heinze, G., Dehghan, M., \& Clase, C. M. (2015). Dietary risk factors for incidence or progression of chronic kidney disease in individuals with type 2 diabetes in the European Union. Nephrology, Dialysis, Transplantation, 30(Suppl. 4), iv76-iv85.

Esposito, K., Maiorino, M. I., Ciotola, M., Di Palo, C., Scognamiglio, P., \& Gicchino, M. (2009). Effects of a Mediterranean-style diet on the need for antihyperglycemic drug therapy in patients with newly diagnosed type 2 diabetes: A randomized trial. Annals of Internal Medicine, 151, 306-314.

Finnish Diabetes Association (2008). Nutrition recommendations for a diabetic. http:// www.diabetes.fi/files/308/Ruokavaliosuositus.pdf (Last accessed 16.2.2016)

Garcia-Fernandez, E., Rico-Cabanas, L., Rosgaard, N., Estruch, R., \& Bach-Faig, A. (2014). Mediterranean diet and cardiodiabesity: A review. Nutrients, 6, 3474-3500.

Gingras, V., Leroux, C., Desjardins, K., Savard, V., Lemieux, S., \& Rabasa-Lhoret, R. (2015). Association between cardiometabolic profile and dietary characteristics among adults with type 1 diabetes mellitus. Journal of the Academy of Nutrition and Dietetics, 115, 1965-1974.

Grosso, G., Stepaniak, U., Micek, A., Topor-Madry, R., Stefler, D., \& Szafraniec, K. (2015). A Mediterranean-type diet is associated with better metabolic profile in urban Polish adults: Results from the HAPIEE study. Metabolism, 64, 738-746.

Hong, X., Xu, F., Wang, Z., Liang, Y., \& Li, J. (2015). Dietary patterns and the incidence of hyperglyacemia in China. Public Health Nutrition, 19, 131-141.

Hsu, C. C., Jhang, H. R., Chang, W. T., Lin, C. H., Shin, S. J., \& Hwang, S. J. (2014). Associations between dietary patterns and kidney function indicators in type 2 diabetes. Clinical Nutrition, 33, 98-105.

Kim, H., Park, S., Yang, H., Choi, Y. J., Huh, K. B., \& Chang, N. (2015). Association between fish and shellish, and omega-3 PUFAs intake and CVD risk factors in middle-aged female patients with type 2 diabetes. Nutrition Research and Practice, 9, 496-502.

Lee, M., Chae, S. W., Cha, Y. S., Cho, M. S., Oh, H. Y., \& Kim, M. K. (2013). Development of a Korean Diet Score (KDS) and its application assessing adherence to Korean healthy diet based on the Korean Food Guide Wheels. Nutrition Research and Practice, 7, 49-58.

Leroux, C., Brazeau, A. S., Gingras, V., Desjardins, K., Strychar, I., \& Rabasa-Lhoret, R. (2014). Lifestyle and cardiometabolic risk in adults with type 1 diabetes: A review. Canadian Journal of Diabetes, 38, 62-69.

Liese, A. D., Bortsov, A., Gunther, A. L., Dabelea, D., Reynolds, K., \& Standiford, D. A. (2011). Association of DASH diet with cardiovascular risk factors in youth with diabetes mellitus: The SEARCH for Diabetes in Youth study. Circulation, 123, 1410-1417.

Lin, J., Fung, T. T., Hu, F. B., \& Curhan, G. C. (2011). Association of dietary patterns with albuminuria and kidney function decline in older white women: A subgroup analysis from the Nurses' Health Study. American Journal of Kidney Diseases, 57, $245-254$.

Nansel, T. R., Haynie, D. L., Lipsky, L. M., Laffel, L. M., \& Mehta, S. N. (2012). Multiple indicators of poor diet quality in children and adolescents with type 1 diabetes are associated with higher body mass index percentile but not glycemic control.Journal of the Academy of Nutrition and Dietetics, 112, 1728-1735.

Reidlinger, D. P., Darzi, J., Hall, W. L., Seed, P. T., Chowienczyk, P. J., \& Sanders, T. A. (2015). How effective are current dietary guidelines for cardiovascular disease prevention in healthy middle-aged and older men and women? A randomized controlled trial. American Journal of Clinical Nutrition, 101, 922-930.

Shai, I., Schwarzfuchs, D., Henkin, Y., Shahar, D. R., Witkow, S., \& Greenberg, I. (2008). Weight loss with a low-carbohydrate, Mediterranean, or low-fat diet. The New England Journal of Medicine, 359, 229-241.

Sievenpiper, J. L., \& Dworatzek, P. D. (2013). Food and dietary pattern-based recommendations: An emerging approach to clinical practice guidelines for nutrition therapy in diabetes. Canadian Journal of Diabetes, 37, 51-57.

Sleiman, D., Al-Badri, M. R., \& Azar, S. T. (2015). Effect of Mediterranean diet in diabetes control and cardiovascular risk modification: A systematic review. Frontiers of Public Health, 3, 69.

Snell-Bergeon, J. K., Chartier-Logan, C., Maahs, D. M., Ogden, L. G., Hokanson, J. E., \& Kinney, G. L. (2009). Adults with type 1 diabetes eat a high-fat atherogenic diet that is associated with coronary artery calcium. Diabetologia, 52, 801-809.

Thorn, L. M., Forsblom, C., Fagerudd, J., Thomas, M. C., Pettersson-Fernholm, K., \& Saraheimo, M. (2005). Metabolic syndrome in type 1 diabetes: Association with diabetic nephropathy and glycemic control (the FinnDiane study). Diabetes Care 28, 2019-2024.

Toeller, M., Buyken, A. E., Heitkamp, G., Cathelineau, G., Ferriss, B., \& Michel, G. (2001) Nutrient intakes as predictors of body weight in European people with type 1 diabetes. International Journal of Obesity and Related Metabolic Disorders, 25, 1815-1822.

US Department of Health and Human Services, National Institutes of Health, National Heart, Lung, and Blood Institute. (1998). Your guide to lowering your blood pressure with DASH. NIH Publication No. 06-4082. Revised April 2006 https:// www.nhlbi.nih.gov/files/docs/public/heart/new_dash.pdf (Last accessed 16.2. 2016)

van Bussel, B. C., Soedamah-Muthu, S. S., Henry, R. M., Schalkwijk, C. G., Ferreira, I., \& Chaturvedi, N. (2013). Unhealthy dietary patterns associated with inflammation and endothelial dysfunction in type 1 diabetes: The EURODIAB study. Nutrition, Metabolism, and Cardiovascular Diseases, 23, 758-764.

Wadén, J., Tikkanen, H., Forsblom, C., Fagerudd, J., Pettersson-Fernholm, K., \& Lakka, T. (2005). Leisure time physical activity is associated with poor glycemic control in type 1 diabetic women: The FinnDiane study. Diabetes Care, 28, 777-782. 